(C2008 IEEE. Personal use of this material is permitted. However, permission to reprint/republish this material for advertising or promotional purposes or for creating new collective works for resale or redistribution to servers or lists, or to reuse any copyrighted component of this work in other works must be obtained from the IEEE. 


\title{
Ontology Based Data Warehouse Modelling - a Methodology for Man- aging Petroleum Field Ecosystems
}

\author{
Shastri L Nimmagadda ${ }^{1}$ and Heinz Dreher ${ }^{1}$, Member, IEEE \\ ${ }^{1}$ School of Information Systems, Curtin Business School, Curtin University of Technology \\ Bentley, Perth,WA, Australia, shastri@eftel.com, h.dreher@curtin.edu.au
}

\begin{abstract}
Petroleum field ecosystems offer an interesting and productive domain for ontology based data warehousing model and methodology development. This paper explains the opportunities and challenges confronting modellers, methodologists, and managers operating in the petroleum business and provides some detailed techniques and suggested methods for constructing and using the ontology based warehouse. Ecologically sensitive operations such as well drilling, well production, exploration, and reservoir development can be guided and carefully planned based on data mined from a suitable constructed data warehouse. Derivation of business intelligence, simulations and vizualisation can also be driven by online analytical processing based on warehoused data and metadata.
\end{abstract}

Index Terms - ontology, data warehouse, data mining, seismic data, petroleum ecosystems.

\section{INTRODUCTION}

Ontology provides semantics, permitting an association among data entities, attributes and even data instances, derived from heterogeneous data sources. Warehouse modelling is logical data integration through multi-dimensional structuring, from different sources of petroleum ecosystems, such as environmental ecology and geomorphology. Environment and geomorphic systems [20, 21, 26] can not be separated and their coexistence is interpreted to be ecologically constructive. This implies that entities involved in these systems are so eco-friendly embedded in nature, but apparently unknown because of their poor understanding. If an entity of a system becomes affected for any reason, this can cause a ripple effect throughout the other systems. For example, geomorphology and its structural pattern are constantly changing on this Earth with a definite impact on its surrounding environment and ecology.

Data from these pertinent systems are inherent in nature. Having known a particular system digitally, the knowledge of associated system can be predicted. For this purpose, these systems, in terms of their digital datasets, are composed intelligently in a data warehouse $[5,7,8,11]$ environment through an ontology modelling approach. Ontology depicts the semantics and the association among these systems, in which entities are represented in multidimensions. Hierarchical and relational ontologies $[4,6,9$, 10] have been described, in which dimensions and their attributes vary in several directions. Ontology builds relationships from fine- to coarse-grained dimensions [13] through bottom-up or up-down hierarchical dimensions. Data attributes of several related entities can also be relationally linked through one-to-many or many-to-many or even one- to-one relationships. Seismic data instances of these attributes are logically organized in vertical, horizontal or lateral directions in relational and hierarchical structuring methodologies. Besides, data instances of dimensions, such as point-to-point, line-to-line, zig-zag and data- looping, can also be structured to integrate different domain ontologies, such as seismic (surface) and well (sub-surface) domains.

Hydrocarbons are explored and exploited from several kilometres of depths of the Earth's crust. Surface excavations, exploited sub-surface structure deformations, petroleum depletion within exploited structures, uneven surface and sub-surface topography and unknown geomorphology are critical issues for successful implementation of well drill plans. Gas leaks and $\mathrm{H}_{2} \mathrm{~S}$ pollutants through chimneys from deep to shallow reservoirs are hazardous for health of a petroleum ecosystem. Oil professionals are concerned with the issues of Environment, Health and Safety (EHS), especially when several hazards are associated with oil/gas field development.

Environment ecosystem and geomorphic systems can not be isolated and are in essence interlinked and interdependent. This natural integrated phenomenon is poorly understood by many explorers. By virtue of the natural integration, one unknown system's characteristics can be predicted from known systems. For example, having known the geomorphic system and its structural pattern, the associated ecological environment surrounding petroleum fields can be predicted. Seismic data instances have been gathered from volumes of 3D seismic survey data, in different dimensions to study and understand the near surface topography. More than 100 wells drilled data and a seismic horizon derived from 230sq $\mathrm{km}$ seismic volume have been integrated and interpreted nearby a matured producing field to study these phenomena.

Building knowledge of geomorphic system and thus predicting an environmental ecology from its associated coexistent interrelated and interdependent systems are key highlights. This study is an attempt to understand systems embedded in nature, to preserve the ecological systems surrounded by matured oil fields and thus extract knowledge from integrated data-warehouse models. This study will facilitate the petroleum managers to optimally design their well-drill plans, preserving the environment and ecosystems.

\section{ISSUES OF Petroleum InduSTRY ECOSYSTEMS}

Quality reservoirs associated with proven entrapped structures [1] produce long and sustained oil and gas production [2]; petroleum and production engineers are responsible in maintaining the health of these quality reservoirs. Surface and subsurface structural deformations [1] always affect the distribution and entrapment of these reservoirs. Geomorphic structures and their interpretation also 
provide sufficient logistics support for these engineers. In our present studies, geomorphic data features are easily interpreted from near-surface seismic data and geological outcrops. Environmental ecology surrounding petroleum fields is predicted using these geomorphic features. An ecosystem surrounding a petroleum field is a system whose entities gain from each other's participation, via symbiotic relationships (positive sum relationships) and referred to as self-sustaining systems. As applied to petroleum industry, an ecosystem is viewed as a system where relationships established across different systems become mutually beneficial, self-sustaining and (somewhat) congested. This is clearly the case for an onshore petroleum industry, operating in an ecologically known geomorphologic system. From a known geomorphology, sustainable environment ecology surrounding a petroleum field is needed for successful well-drill plans. Once ecosystems and their embedded inheritances are established, the costs are calculated in a more optimised way. This embedded ecosystem has significance for exploring similar ecosystems that can interconnect the present systems. This will drastically lower the costs of well-drilling in the operational areas.

Ecology, narrating the environment surrounding a petroleum field, guides suitable places for exploration drilling. Sinkholes are hazardous areas for exploration and drilling. Plan and design of underground hydrocarbon storage tanks are significant in areas of sinkholes, which need to be addressed carefully [27]. High pressure areas, if associated with suspected shallow sinkholes need to be monitored, especially production facilities $[26,28,29,31,33] . \mathrm{H}_{2} \mathrm{~S}$ pollutant areas are to be carefully examined [29], if they are connected with surface exposed faulted structures. Exposure of $\mathrm{H}_{2} \mathrm{~S}$ is dangerous and these areas are to be examined, if they are, by chance, linked with sinkholes and or gas chimneys. Authors attempt to explore these hazardous areas using latest IT and telecommunication technologies $[1,6-14]$ in the producing areas. Ontology based data warehouse modelling and data mining approaches are proposed to address these issues. Seismic geophysics and welllogging survey data entities, attributes and their data instances are intended to be used in the present studies. Sinkholes also pose serious problems to the plan and design of perforation intervals $[26,30,32,34]$. Wells may not have been successfully completed because of these issues. The authors attempt to organize seismic and well datasets in multidimensional data structuring and map them for datamining and exploration for hazardous areas.

It is frequently the case in petroleum producing areas that sedimentary rocks are surface-exposed. When an underground cavity enlarges (especially in karsts or carbonate environment) to the point that its ceiling can no longer support the weight of overlying sediments, the earth suddenly collapses into the cavity. A circular hole (so called sinkhole) typically forms and grows over a period of minutes to hours. Slumping of the sediments along the sides of the sinkhole may take approximately one day to stop. Erosion of the edge of the sinkhole may continue for several days, and heavy rainfall can destabilize environment around sink- hole. In the less catastrophic cover subsidence type of sinkhole, a bowl-shaped depression forms at the surface, typically over longer periods of time (sometimes as long as years). Probable triggering mechanisms for sinkhole collapse may include, overburden pressure, blasting, heavy ground loading, heavy rainfall, and heavy ground-water pumpage.

Professional geologists and geotechnical engineering consultants perform a variety of tests to attempt to locate buried cavities which might have formed due to sinkholes. These tests include ground penetrating radar surveys, electrical resistivity tests, and borings. However, test results may be affected by the local geology and elevation of the water table, and are not always conclusive. Seismic reflection survey, though expensive, are useful in exploring sink holes, especially in surrounding petroleum producing matured fields to avoid disastrous environmental consequences. Seismic reflection surveys are capable of investigating the complete package of shallow as well as deep sinkholes.

Maintaining a database, documenting all past sinkholes is good idea. This represents only those sinkholes officially reported by observers. The reported sinkholes tend to cluster in producing areas where they are readily seen and commonly affect shallow geomorphology. However, numerous sinkholes also occur in fields, many of which remain unseen and unreported. Sinkholes are a common feature of a landscape system. They are only one of many kinds of karsts landforms, which include caves, disappearing streams, springs, and underground drainage systems. "Karst" is a generic term, which refers to the characteristic terrain produced by erosion processes associated with the chemical weathering and dissolution of limestone or dolomite, the two most common carbonate rocks. Dissolution of carbonate rocks begins, when they are exposed to acidic water. Most rainwater is slightly acidic and usually becomes more acidic as it moves through decaying plant debris. Limestone rocks are porous, allowing the acidic water to percolate through their strata, dissolving some limestone and carrying it away in solution. Over eons of time, this persistent erosion process may have created extensive underground voids and drainage systems in much of the carbonate rocks surrounding petroleum fields. Collapse of overlying sediments into the underground cavities produces sinkholes. Dry caves are parts of karst drainage systems that are above the water table.

\section{ONtology-base Warehouse Modelling METHODOLOGY}

Examination of sinkholes and their formation depend on availability of geological and geophysical data. How these sinkholes can be detected from reflection-base seismic data are discussed in this section. Theoretically, seismic data distort their amplitudes and frequencies with possible loss of seismic energy surrounding sink-holes where severe absorption effects take place. Here, seismic data from different domains are logically organized and integrated from seismic to well datasets. An ontology-based approach has 
been discussed to conceptualize the entities involved in seismic and well domains. Geomorphic structural patterns are computed using these ontologically derived data structures.

\section{A. Ontology-based conceptual modelling}

Volumes of dimensions are conceptualized and generate ontology-based multidimensional data models, building relationships among dimension and fact data tables of seismic and drilled-well entities. Where ever one-to-one, one-tomany and many-to-many relationships exist they have been mapped more logically. Business rules, constraining the data mapping process, have been imposed while building relationships among data structures.

\section{B. Functional Mappings in Seismic Domain Ontology}

Most often in multidimensional ontology modelling [3, $6,7,8,9,10,11,12,13]$ different dimensional data are dealt with. These data containing several entities and attributes are conceptualized to build ontology relationships. In discrete theory of datasets and relations [16], a relationship between two domain ontologies defines a correspondence, or connection, or mapping among elements of datasets. These datasets can consist of numerals and symbols as in mathematics or entities in databases. A relation [16] is an ordered pair of elements taken from the related domain ontologies. In other words, one element from one dataset of particular domain ontology can be combined with one from the other dataset of a related domain. In our seismic data instances case, the relationships are often of functional type, in which the mapping structure of a relationship (between seismic and well-base domains) determines the type of a relationship (as shown in Figs. 1a and 1b) and also obeys the mathematical definition of a function.

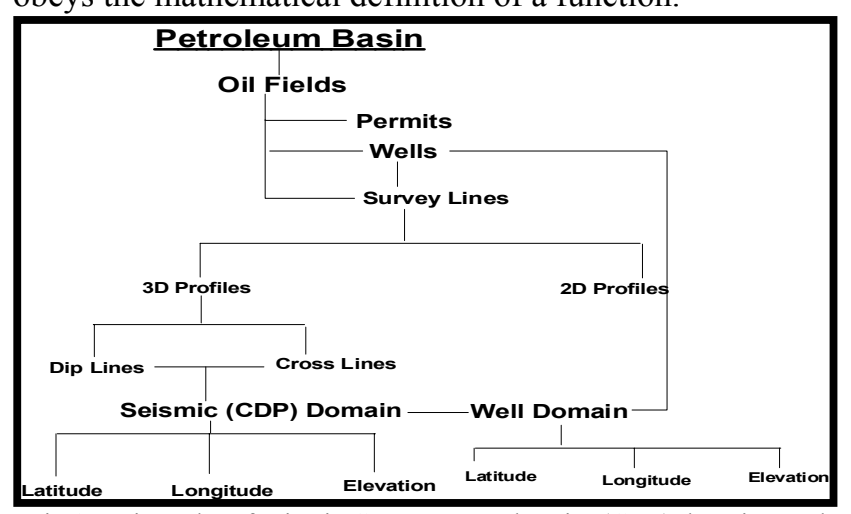

Fig 1a: Hierarchy of seismic Common Depth Point (CDP) domain ontology, showing different varying data dimensions

A special type of mapping [16] that is of great importance in ontology is the mapping that obeys the mathematical definition of a function. In a mathematical function, a domain $\mathrm{D}$, a range, $\mathrm{R}$, and a functional mapping or function $f$ such that $f: D \rightarrow R$, which means that for all values in each domain ontology, D map to the values of other related ontology $\mathrm{R}$, such that for each value in $\mathrm{D}$, there corresponds a unique value in $R$. If every value in $D$ maps to $R$, then it is totally "functional"; otherwise the mapping is called partially functional. In our case, seismic data instances, all the domains, specified, such as time, velocity and depth, every value in each domain, maps every value in the other domains. So these are totally functional mappings.

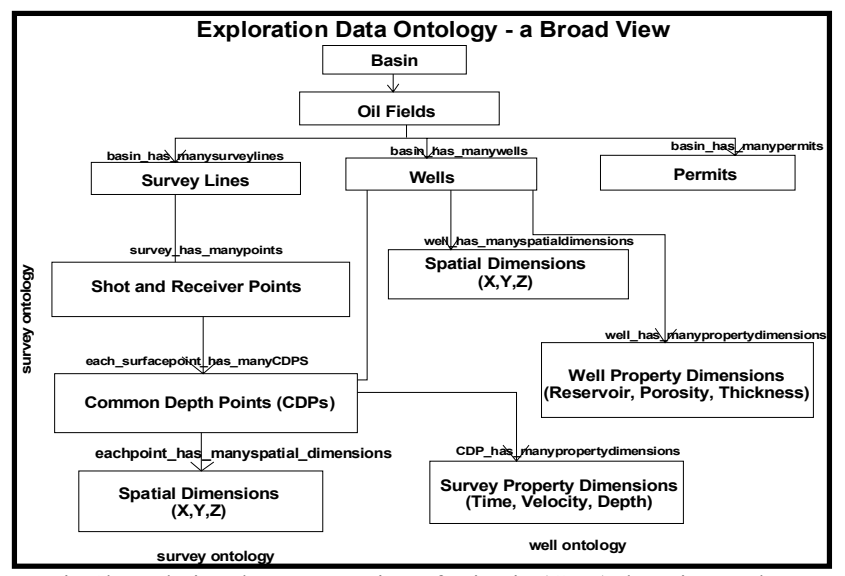

Fig 1b: Relational representation of seismic (CDP) domain ontology, showing different varying data dimensions

Hierarchical databases have their idiosyncrasies [16]. Some are useful for applications, such as functional mapping between parent and children (Fig.1) and the hierarchical structure. Hierarchies pose restrictions. To be more specific, in a hierarchy or tree data structure, there is a manyto-one mapping (which includes one-to-one) from child nodes (data record instances) to the root instance. This must be a totally functional mapping. The hierarchy must obey the tree structure in the sense that that no node can exist without a parent except the root. For example, "petroleum basin" and "exploration" entities must have their related sub-type entities or dimensions (as shown in Fig. 1). Further, either one or more attributes must have been related among these dimensions in a deduced hierarchy. The hierarchy may have several applications too, where for example, children need to be unassigned to any parent; handle the deletion of a parent differently; and build relationships of a more complex nature such as many-to-many relationships or the multidimensional relationships ending up in a lattice or network structure rather than a tree structure.

The exploration entity or dimension has a complex hierarchical data structure, represented in a multidimensional conceptual data model. In case of seismic and well-data structuring, for example, 3D seismic data are distributed in multi-dimensions, each dimension characterizing a property. In case of hierarchical structure, such dimensions are either mapped vertically or horizontally or laterally and in combinations. These ontologically derived conceptual entities are further translated into different multidimensional data properties for intelligently storing in a warehouse environment, and discussed in the following sections.

\section{Data Structuring - Hierarchical Ontology}

In hierarchical data structuring [13, 14], hierarchies of geomorphic structural entities are constructed. A hierarchy is a tree structure, in which a geomorphic surface, as represented by seismic dimension has several nodes and each node is connected by lines, points and contours and all these nodes are interconnected. Each geomorphic surface consists of number of contours, each contour has equal data 
instance values (such as time, depth and velocity values) that are distributed on several survey grids and on each grid with several survey lines and on each line with several points. Each point has latitude, longitude and elevation as spatial dimensions along with property instance. These are typical hierarchical data structural features. In our case of hierarchical data organization, conceptual ontologies are built among several geomorphic surfaces surrounded by petroleum fields. A profile layout describing generalized hierarchical and relational seismic domain ontology is shown in Fig. 2. One such surface that is representing the near surface geomorphic features is discussed in the following sections.

\section{C.1. Vertically varying dimensions}

Vertically varying dimensions also called, vertical dimensionality have time, velocity and depth are key dimensions, varying their instances hierarchically in the vertical direction. When their instance values vary in this direction, the data structure changes into different relationship or other conceptual domains, such as slopes (steepness dimension) of horizons. The strike and dip attributes (their respective instances) of horizon dimensions thus come into play. Entities participating in this type of data structuring, may be top-down from coarse grain to fine grain (until it is mapped to its atomic level) or down-top from fine to coarse (from denormalization to normalization) structuring approach.

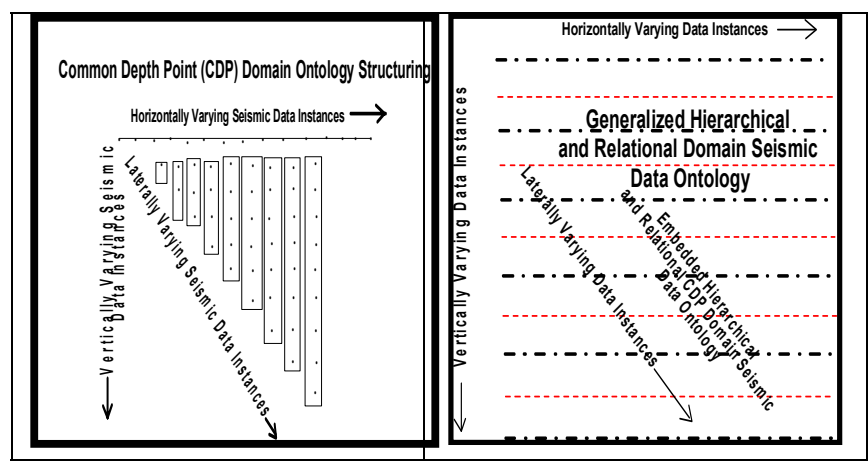

Fig. 2: 3D Profile layout describing generalized hierarchical and relational seismic domain ontology

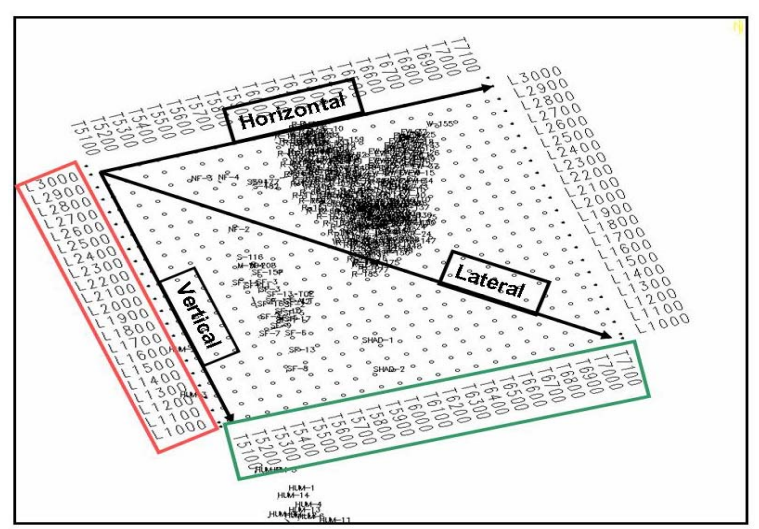

Fig. 3: Survey areas with spatial dimensions - analysing the hierarchical and relational ontologies for geomorphic representation

As shown in Fig.3, horizontal, vertical and lateral dimensions are extracted and described in relation to the hier- archical data structuring. Horizontal dimension is typically a space; vertical dimension is either time or depth; and lateral may be a composite dimension, comprising both space and time or depth dimensions. Line may be a composite dimension in the horizontal, vertical and lateral directions. These are further described in the following sections.

\section{C.2. Horizontally varying dimensions}

Each layer discussed in hierarchical data structuring, has distinct dimension attributing to a certain property. Points, survey lines under seismic domains are represented each in different data layers in horizontally changing hierarchies.

\section{C.3. Laterally varying dimensions}

In case of laterally varying hierarchies, data layers may be conceptualized. For example, in a geomorphic structured surface, may have several data grids, from 2D and 3D survey lines, having multiple data instances gathered from laterally changing dimensions.

\section{Relational Data Structuring-Relational Ontology}

Relational data structuring is based on the relational model [3], in which data may have internal conceptual relationships, describing relations among several attributes, called relational-base ontology. Several datasets in a domain make up a relation. A significant feature of relational data structuring is that attribute values come from a homogeneous pool of values called a domain, which represents all possible values in a finite dataset. However, more than one attribute can assume values from the same domain and a single attribute can take several values from a domain. In the seismic data structuring case, single "seismic" domain ontology contains time, depth and velocity dimensions and their instances contributing to describe a "time surface". This represents a geomorphic surface, narrating several structural features.

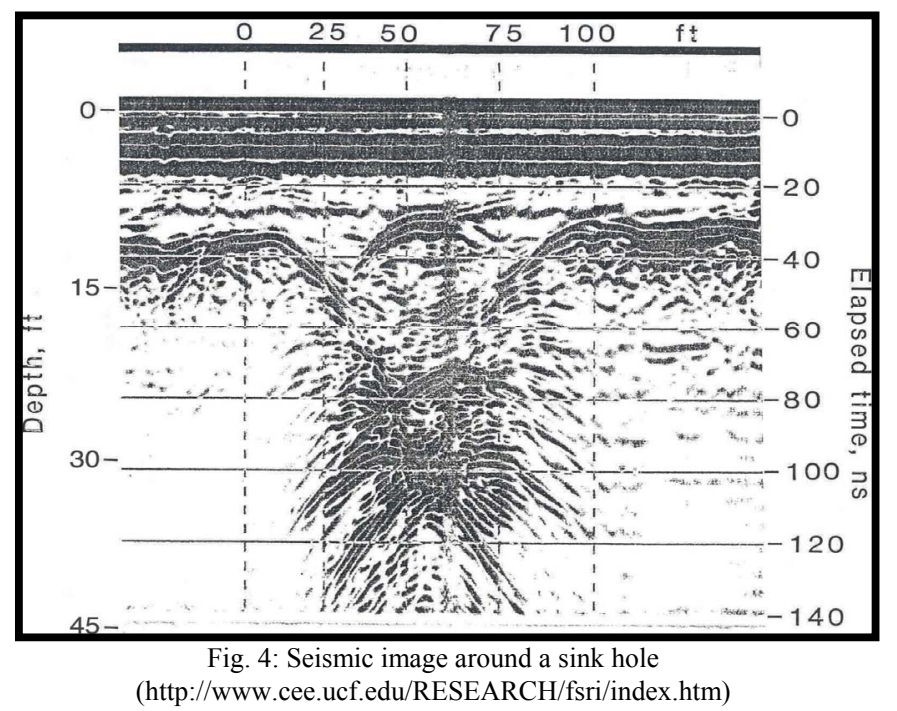

As shown in Fig. 4, one can visualize the impression or image of a typical sink hole, which describes multiple dimensions such as depth, time and space. Lateral dimensions can also be extracted from these digitally recorded data. 
Contoured surface has alignment of equal time, or depth or velocity data values, taken from several in-lines and crosslines, with points; each point has a property value. As stated earlier, each point consists of several dimensions. These dimensions have been pictorially described in the Fig. 5.

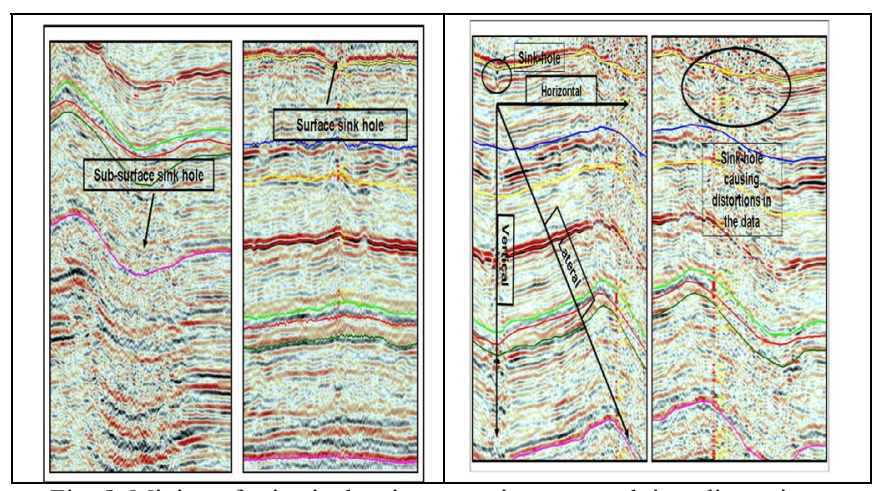

Fig. 5: Mining of seismic data instances in space and time dimensions depicting signatures of sink-holes, causing distortions in the seismic amplitudes and frequencies

Sink holes have been noticed or interpreted on shallow surfaces as shown in Fig. 5, affecting deeper sections of the seismic data. The data instances from these dimensions have been extracted from the digitally recorded data.

\section{D.1. Vertically varying data relationships}

The most common dimension, varying its instance in vertical direction is either time or depth. Either time or depth has definite relationship with geomorphic structure attributes. From seismic times, depth relationship is built using another relationship, called velocity. Velocity is a key dimension attributing to depth variations in vertical direction. Utmost care is necessary to interpret seismic data instances along their survey grids. Having generated contoured surfaces at chosen time or depth, one can interpret for geomorphic data features. Other dimensions changing in vertical direction are seismic amplitude, frequency and phase attributes. Knowledge of geomorphic surface structure depends on interpretation these vertically varying data attributes.

\section{D.2. Horizontally varying data relationships}

Most common dimension, attributing to changes in the horizontal direction, is spatial dimension, with a distance property, separating two points on a surface. Distance between points has a definite relationship with geomorphic structure properties. Horizontal variations in seismic times, depth or velocity dimensions and their attributes are interpreted to be associated with conceptual structural variations in geomorphic surface features.

\section{D.3. Laterally varying data relationships}

The relationships of data dimensions, built laterally are conceptual. Ontologically, all these data instances are conceptually described and interpreted along these changing seismic data structural features. As shown in Fig.3, the survey location map, representing positional or coordinate data, narrates different domains of ontology in which surveys and wells are located. There data instances are common in these different domains. Relationships are conceptualized wherever they need to be built for the purpose of extracting knowledge of hazards on the geomorphic structured surfaces.

\section{E. Warehouse Modelling of ontologically derived in- stances}

Changes in geomorphology are causative to impacts on environmental ecology because these systems are interconnected in nature. This phenomenon is well understood in a data warehouse modelling situation (a framework as described in Fig. 6), integrating the datasets of geomorphic structures and other associated geological outcrop features. Data are gathered and organized intelligently from different levels of hierarchies in both horizontal and vertical dimensional structuring process. All the dimension tables (with rows and columns) with their corresponding fact tables are intelligently stored using normalization process. For fine grained data structuring purposes, at places, relationships are denormalized among several dimension and fact tables.

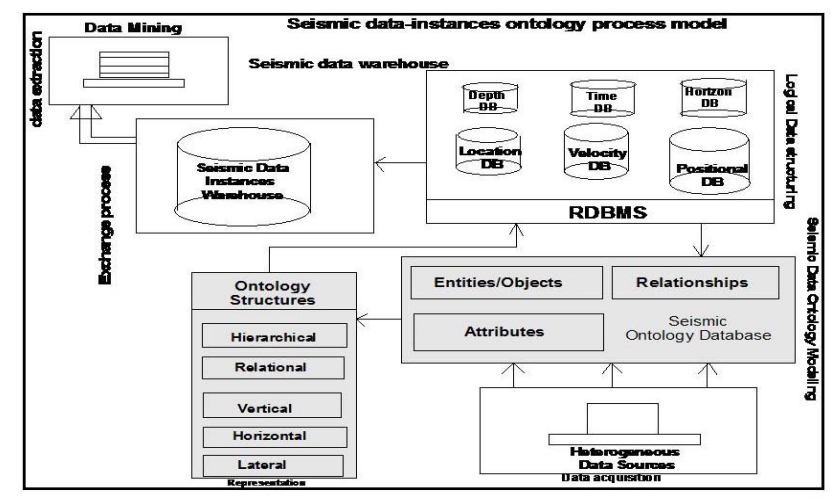

Fig. 6: Framework of warehousing seismic data instances

Besides, time, depth, location, horizon and positional dimensions (Fig. 6), dip and strike dimensions are significant to interpret the horizon, from which all the associated relationships are correlated and mapped respectively in horizontal and vertical point-to-point and dip tracking dimensions. One can take advantage of the fact that multiple dimensions existing in exploration entity $[9,10]$ can effectively be used for building conceptual data models and integrated into metadata for extracting knowledge, especially between seismic and well-data domains. Fig.6 is basic framework used to construct ontology based multidimensional data structure maps as interpreted in Figs. 9-10.

Data integration, business data intelligence [17], repository and modelling are key aspects of metadata development. Business continuity is a key issue in the data integration process, meaning that exploration and production data are distributed from different applications and each application is handled by a single operational unit (such as field development, exploration, drilling, production etc). Data gathered from different domains across many operational units of organization go into a warehouse to enable access and retrieval by many users and to extract business knowledge using online analytical tools. Having integrated differ- 
ent domain of data, validity of information extracted from the data warehouse is analysed through synchronization of distributed data and information. A consolidated data integration platform should support data consistency management requirements across a broad, diverse set of DBMSs, operational platforms, and data types. Ontology supports $[18,19]$ designing metadata and development of a data integration (Fig. 7) process since conceptual models of different domains are intelligently integrated and stored in a warehouse.

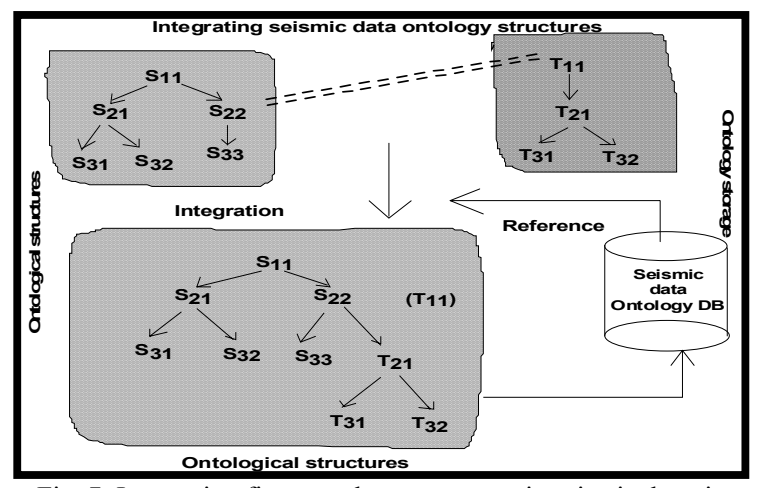

Fig. 7: Integrating finer ontology structures in seismic domain Source: adapted from [9]

Logically organizing the seismic and well data instances in the form of metadata is an art of data representation, in which metadata serves to identify the contents and location of these datasets in a warehouse. A warehoused seismic and well-base metadata repository should consist of:

1. Description of structure of the data warehouse. This includes the warehouse schema, view, dimensions, hierarchies and derived data definitions, data locations, and contents;

2. Operational metadata, such as relationships, conceptualizing the dimensions, data units and monitoring information on seismic and well data qualities with updates;

3. The summarization processes which include dimension definition, data on granularity, partitions, summary measures, aggregation and summarizations;

4. Details of data sources, which may include source databases and their contents, gateway descriptions, data partitions, data extractions;

5. Data related to system performance, which include indices and profiles that improve data access and retrieval performances, in addition to rules for timing and scheduling of refresh, update and replication cycles;

6. Business metadata, which include business terms, rules, constraints and definitions, data ownership and changing policies on Environment, Health and Safety (EHS).

In our case, we have considered seismic, well-data and other available geological inputs for ontology-based warehouse modelling. More specifically, an objective to extract knowledge through ontology structuring and integration of different domain data is focused upon. Different dimensions described in each entity have been structured through multidimensional knowledge-base data structuring. Besides seismic time, velocity (geophysical dimensions) and formation depth (geological dimension) dimensions, structure dimension with "structure-high" and "structure-low" attributes (Fig. 5). Type of karsts, volume of karsts, which subside producing geological formations and liquefied media, which leach the karsts, have definite impacts on amplitude and frequency content of seismic data instances. This has close relationship with conceptualized "structure" attributes. Karst terrains represent unique characteristics of seismic data instances along dip dimension and they are different when data instances are represented along strike dimension. The attribute properties change as per change in geological dimensions.

\section{Results AND Discussions}

A data warehouse is a computer system designed for archiving and analysing an organization's historical data, such as exploration and production data, drilling data, or other information from day-to-day operations. Normally, an organization summarizes and copies information from its operational systems to the data warehouse [23, 24, 25] on a regular schedule, such as every night or every weekend; after that, management can perform complex queries and analysis on the information without slowing down the operational systems. Data integration is key issue; combining data residing at different sources and providing the user with a unified view of these data. This important problem emerges in a variety of situations both commercial (when two similar companies need to merge their databases) and scientific. Data integration appears with increasing frequency as the volume and the need to share existing data increases. Preparation of data warehouse to Data Mining (DM), also called Knowledge-Discovery in Databases (KDD) or Knowledge-Discovery and Data Mining, is the process of automatically searching large volumes of data $[17,23]$ for patterns and deriving association rules. This is a fairly recent topic in computer science but applies many older computational techniques from statistics, information retrieval, machine learning and pattern recognition.

In our present study, initially all the data entities are conceptualized into ontology-based multidimensional structuring. Then relationships are built based on ontology modelling are integrated in a warehouse environment. Various data views extracted from the warehouse are interpreted for building knowledge from warehoused metadata. Seismic and well data, thus represented into metadata, have been interpreted for geomorphic features and then predict environment ecology around matured producing fields.

\section{F. Ontologically interpreted seismic data volumes}

Warehoused data are mined for interpreting data views in terms of geology. Karst terrains develop in areas underlain by carbonate rocks such as limestone rocks. They often have drainage systems that are reflected on the surface as sinkholes, springs, disappearing streams or even caves. The term "karst", therefore, refers to the terrain and the term sinkhole is one of the types of drainage features reflected 
by that type of terrain.

In our data warehouse modelling approach, seismic data instances from hierarchical and relational structuring in both horizontal and vertical dimensions are gathered and intelligently stored. From different data mining representations of seismic data instances (in time and depth domain) as shown in Fig. 5, demonstrate several geomorphic features in which sink holes are represented by structural lows. These structural lows, unlike structural anomalies, have unusual shapes and patterns. Amplitude maps and formation thickness maps (subtraction from formation top with formation base), narrate shallow drainage patterns and also strength of karstification. Kastification can be visually seen as described in Fig. 8, in which several mine fields have also been reported in the seismic grids (left image of Fig. 8).

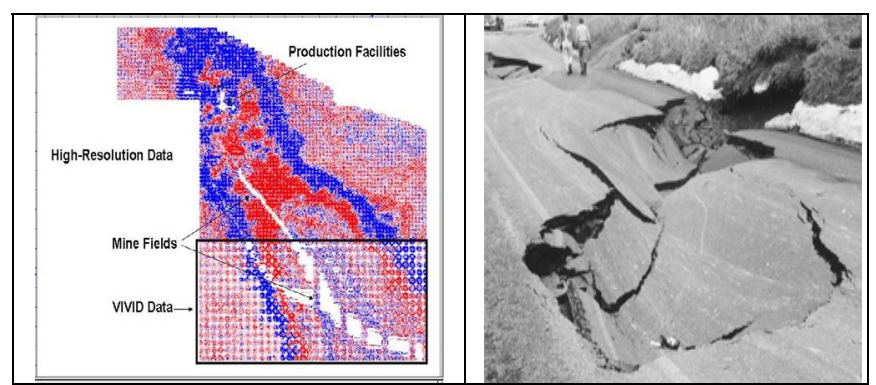

Fig. 8: Seismic time dimensions, showing data variations across minefields in the survey area - a scenario associated with geomorphic system (right image)

Near surface features such as data acquisition layout foot-prints, mine-fields, production facilities can clearly be visualized in high resolution and sparse data grid areas as shown in Fig.8.

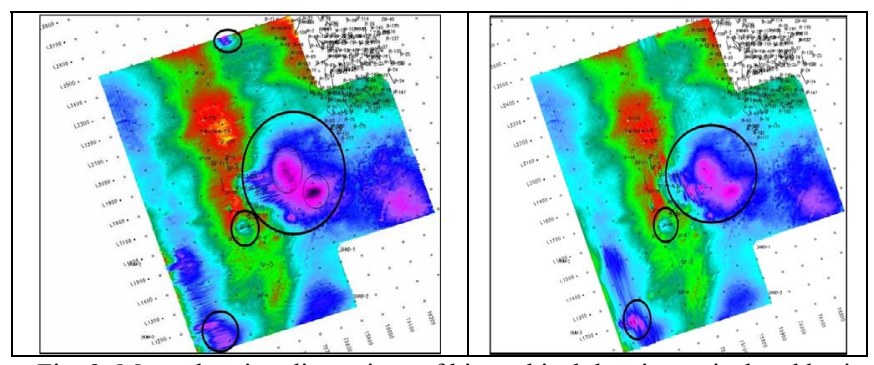

Fig. 9: Maps showing dimensions of hierarchical data in vertical and horizontal directions - sinkhole signatures of geomorphic representation

There are many oil producing wells in the areas from carbonate reservoirs in the study area. As stated earlier, carbonate rocks when associated with liquefied media cause chemical reactions to take place thus creating cavities, voids and even fractures with carbonate rocks. These anomalies definitely must have deformed the rock properties which have been represented as sinkholes as shown pinkish circular features in Fig. 9. Signatures due to sinkholes (encircled blue bodies) are representative of loss of seismic energy and dissipation of seismic amplitudes and frequencies seen in the form of circular features as shown in Figs.9-10. The framework described in Fig 6, is used for constructing integrated Metadata structures and interpreting them into multidimensional data structure maps as narrated in Figs. 9-10.
The relationship has been built based on integration of data instances extracted from seismic and well-base data structure domains. Structure attributes, represented as "structure-highs" (red colour notation) and "structure-lows" (blue coloured notation) as shown in Figs. 9-10 are anomalous features, are interpreted for geomorphologic structure patterns. These sinkholes, which are seen in the seismic data as structural low anomalies, will manifest environmentally as geomorphic structure features shown in Fig. 10.

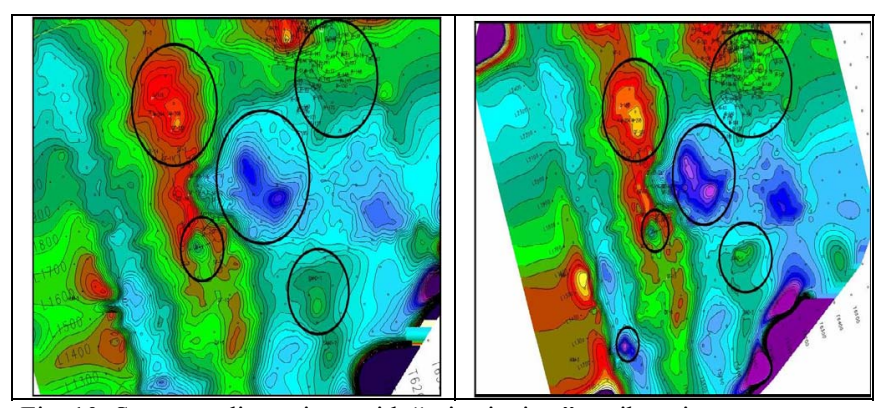

Fig. 10: Structure dimensions with "seismic time" attribute instances contoured over a survey over a geomorphic representation

The real difference between actual depositional structural low and sinkhole structural low, is that depositional structural low is broader and gently dipping towards depocentre, whereas sinkhole structural low has more sharp edges with steep dips. As shown in Figs 9-10, where one can physically interpret sharp edge with steep cut. Though the sinkholes described in the seismic images do not correspond to the sinkholes demonstrated in Fig. 11, but ultimately, geomorphic features discussed in Fig.10 will take shape of the image as shown in Fig.11.

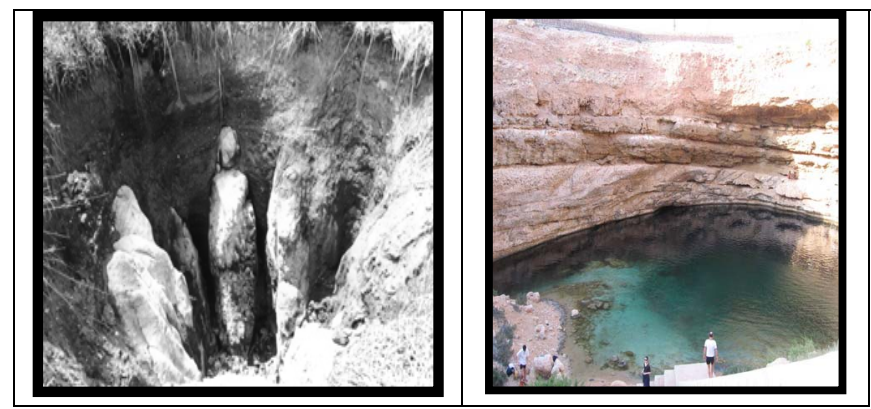

Fig. 11: Sink holes affecting petroleum ecosystem (known sink-holes from: http://www.sinkhole.org/facts.htm)

\section{G. Conclusions and Recommendations}

Ontology provides necessary semantics and contextual mapping among several petroleum data entities. Ontology is recommended for better conceptual and relationships among entities associated with geomorphology and environmental ecology surrounding matured or developed petroleum fields. Data warehousing accommodates an integrated ontology in the form of multidimensional metadata structuring. Ontology based data warehousing has immense value at the stage of data mining, especially knowledge mapping among several operational data entities in the petroleum industry. Fine grained multidimensional data structuring is an asset for knowledge building in different operational domains, in particular exploration, drilling and pro- 
duction stages. The authors have attempted to visualize and interpret sink-holes on near surface time and depth domain structure maps, in particular shallow sink-holes. However shallow sink holes (circular blue bodies encircled in Figs. 9-10) have definite impact on deep seated sink-holes, which have been also mapped on deeper seismic horizons in work in progress which also connects several outcropped and surface-mapped sink-holes through seismic and drilling programs.

These studies are useful for well-drill planning and also preserve ecological systems. Professionals involved in the petroleum exploration, drilling and production operations, are recommended to use these concepts and commission them appropriately in the petroleum field development. Structure and reservoir models prepared in these ecologically disturbed areas provide good clues where future drilling is planned, both for development and exploration wells.

\section{REFERENCES}

[1] Chakraborty, K. Mubarak, Al. H, Nimmagadda, S.L. and Ray, J. "Exploration Data Integration, an effective reengineering process for new petroleum plays in Gulf Offshore Basins", presented at the 2006 International Conference of $A A P G$, November, Perth, Australia.

[2] Gilbert, R. Liu, Y. Abriel, W. and Preece, R. "Reservoir modeling: integrating various data at appropriate scales", Leading Edge (2004), Volume 23 (8), p.784-788.

[3] Hoffer, J.A, Presscot, M.B and McFadden, F.R. "Modern Database Management", (2005), $7^{\text {th }}$ Edition, Prentice Hall.

[4] Jasper, R. and Uschold, M. A. "Framework for understanding and classifying ontology applications", published in the proceedings of the IJCAI-99 ontology workshop, p.1-20.

[5] Jukic, N. and Lang, C. "Using offshore resources to develop and support data warehousing applications", Business Intelligence Journal (2004), p.6-14.

Lowrie W: "Fundamentals of Geophysics". New York, New York, USA: Cambridge University Press, 1997.

[6] Nimmagadda, S.L. and Dreher, H. "Ontology of Western Australian petroleum exploration data for effective data warehouse design and data mining", presented and published in the proceedings of the $3^{\text {rd }}$ international IEEE conference on Industrial Informatics, (2005), Perth, Australia.

[7] Nimmagadda, S.L. and Dreher, H. "Data warehouse structuring methodologies for efficient mining of Western Australian petroleum data sources", a paper presented and published in the Proceedings of $3^{\text {rd }}$ international IEEE conference on Industrial Informatics, (2005), Perth, Australia.

[8] Nimmagadda, S.L, and Dreher, H. "Mapping of Oil and Gas Business Data Entities for Effective Operational Management", presented and published in the proceedings of the $4^{\text {th }}$ International Conference of IEEE Industry Informatics, (2006), Singapore.

[9] Nimmagadda, S.L. and Dreher, H. "Ontology-Base Data warehousing and Mining Approaches in Petroleum Industries": in Negro, H.O., Cisaro, S.G., and Xodo, D., (Eds.), Data Mining with Ontologies: Implementation, Findings and Framework, a book published in 2007 by Idea Group Inc., http://www.exa.unicen.edu.au/dmontolo/
[10] Nimmagadda, S.L., and Dreher, H. "Ontology based data warehouse modelling and mining of earthquake data: prediction analysis along Eurasian-Australian continental plates", a paper accepted for presentation in the International Conference of IEEE in Industry Informatics Forum, (2007) Vienna, Austria.

[11] Nimmagadda, S.L. and Rudra, A. "Applicability of data warehousing and data mining technologies in the Australian resources industry", presented at the 7th international conference on IT, (2004), Hyderabad, India

[12] Nimmagadda, S.L. and Rudra, A. "Data sources and requirement analysis for multidimensional database modeling - an Australian Resources Industry scenario", presented at the 7th international conference on IT, (2004), Hyderabad, India.

[13] Nimmagadda, S.L, Dreher, H. Chang, E. and Rajab, M.R. "New technologies in mature gulf basins - multidimensional modelling of ontologically derived historical petroleum exploration data properties for effective basin knowledge mapping", a poster paper presented and published in the proceedings of $A A P G$ international conference and exhibition, (2006), 5-8 November.

[14] Nimmagadda, S.L, Dreher, H. and Rudra, A. "Warehousing of object oriented petroleum data for knowledge mapping", a paper presented and published in the Proceedings of the $5^{\text {th }}$ International Conference of IBIMA, (2005), Cairo, Egypt.

[15] Opdahl, A.L., Henderson-Sellers, B., and Barbier, F. "Ontological analysis of whole parts relationships in OO models", Information and Software Technology, (2001), 43, pp. 387-399.

[16] Ozkarahan, E. "Database Management, Concepts, Design and Practice”, (1990), Prentice Hall Publications.

[17] Pujari, A.K. "Data Mining Techniques", (2002), Universities Press (India) Private Limited, p 1-40.

[18] Shanks, G. Tansley, E. Weber, R. "Using Ontology to validate conceptual models", communications of the ACM, (2003), 46(10), pp. 85-89.

[19] Shanks, G. Tansley, E. and Weber, R. "Representing composites in conceptual modelling", Communications of ACM, (2004), 47 (7), pp. 77-80.

[20] Sheriff, R.E, Oliveira, N. E. D. "Dictionary of Applied Geophysics", (2002), $4^{\text {th }}$ Edition, SEG Publishers, p. 1-429.

[21] Telford, W.M. Geldart, L.P. and Sheriff, R.E. "Applied Geophysics", (1990), Second Edition, p.100-350 and 600-750.

[23] Uschold, M.E. "Knowledge level modeling: concepts and terminology", Knowledge Engineering Review, (1998), 13(1).

[24] Uschold, M. and Gruninger, M. Ontologies: "Principles, methods and applications", Knowledge Engineering Review, (1996), 11(2).

[25] Wand, Y., Storey, V.C., and Weber, R. "An ontological analysis of the relationship construct in conceptual modelling", ACM Trans. On Database Systems 24 (4), (1999), pp. 494-528.

[26] Electronic Newsletters on the Petroleum and Utilities Industry; http://www.api.org/ehs/

[27] Closure of Underground Petroleum Storage Tanks - API RP 1604, $3^{\text {rd }}$ Edition, p 17, 1996

[28] Rolf K. Eckhoff, Explosion Hazards in the Process Industry, API Publication, p. 441, 2005.

[29] H2S Safety Handbook, PETEX Publications p.16, 1992.

[30] Longley, M, Analysis for Well Completion, PETEX Publications, p. 108

[31] Health Safety and Environmental Reference Guide, IADC Publications, p.192, 2004

[32] Luiz Otávio Amaral Affonso, Machinery Failure Analysis Handbook, Gulf Publishing Company, p.300, 2006

[33] Hanson, M, Implications for Environment and Safety Management, TSCI, http://www.tsci-hse.com:8080/ layouts/tsci/default.aspx 